\title{
Benign extramedullary myeloid proliferations
}

\author{
Dennis P O’Malley \\ US Labs, Irvine, CA, USA
}

\begin{abstract}
Extramedullary proliferations of bone marrow elements are infrequently encountered in routine pathology practice. On occasion, they can present diagnostic difficulties when seen in unusual or unanticipated sites. This review serves to cover aspects of underlying embryogenesis of myeloid elements, as well as sites and circumstance of benign proliferations of myeloid elements along with their occasional confusion with neoplastic myeloid proliferations. Benign proliferations associated with hematologic disorders and hematopoietic growth factors are discussed. Immunohistochemical evaluation of myeloid proliferations is considered as well.
\end{abstract}

Modern Pathology (2007) 20, 405-415. doi:10.1038/modpathol.3800768; published online 2 March 2007

Keywords: extramedullary hematopoiesis; spleen; lymph node; myelolipoma; myeloid sarcoma; diagnosis

The production of blood cells by the bone marrow is the basic function of the hematopoietic system. The appropriate proliferation and production of marrow elements is fundamental to the control of numerous body systems, because of the far-reaching effects of the hematologic elements. Oxygen transport by red cells, coagulation by platelets and the nonspecific immune responses by granulocytes and monocytes are the essential functions, but there are numerous other, more subtle functions of the hematopoietic system as well.

The embryologic development of the hematopoietic system is exceedingly complex and relies on close interaction with other systems of the body. The delicate orchestration of development can be seen as the primary site of hematopoiesis changes from yolk sac to liver and finally within the marrow cavity.

Under normal circumstances after birth, there is little proliferation of hematopoietic elements outside the marrow. However, in a variety of pathologic conditions, marrow elements will be present, and sometimes proliferate, outside the relatively specific environment of the bone marrow. Myeloid proliferations have been reported in a wide variety of sites including lymph nodes, mediastinum, breast, central nervous system, peripheral nerves, orbit, middle ear, pancreas, urethra, oropharynx, nasopharynx, pleura and lungs, pericardium, heart, gastrointestinal tract, peritoneum, thyroid gland, skin, subcutaneous tissue, kidney, adrenal gland, prostate gland,

Correspondence: Dr DP O’Malley, MD, US Labs, 2601 Campus Drive, Irvine, CA 92612, USA.

E-mail: domalley@uslabs.net

Received 20 October 2006; revised 03 January 2007; accepted 17 January 2007; published online 2 March 2007 epididymis, ovaries and endometrium. ${ }^{1,2}$ It is these circumstances and the proliferation of marrow elements in extramedullary sites that are the focus of this review. Evaluation of neoplastic myeloid proliferations (NMP), in the context of differential diagnosis, will also be briefly discussed.

\section{Benign}

Embryology

To understand better the development of extramedullary hematopoiesis (EMH), a brief review of normal hematopoietic development is necessary. The derivation and ultimate construction of an intact hematologic system from the embryo to its adult form is a complex process with many steps and numerous molecular and cellular interactions. In the earliest stages of embryo development, the yolk sac is the earliest site of primordial development of the hematopoietic system. ${ }^{3}$ Aggregates of mesodermal cells condense and form vascular elements within the yolk sac. Clusters of primitive mesodermal cells that develop into hematopoietic cells develop in close association to these blood vessels and are termed blood islands. These early hematopoietic elements begin to circulate in the embryo as soon as the vascular elements allow (approximately 20 days). In parallel to development of hematopoiesis in the yolk sac, the rudimentary liver develops. In the fetal liver, hematopoietic progenitor cells are not produced, but are colonized by circulating hematopoietic precursors, which proliferate and differentiate in the liver parenchyma. At approximately day 30 , the liver is the primary site of hematopoiesis (but not generation 
406

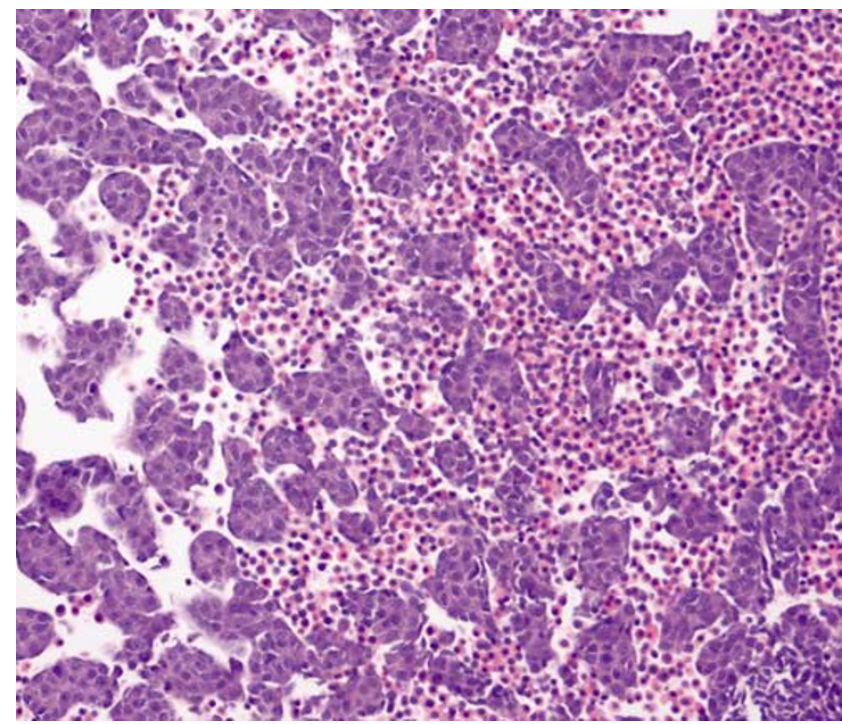

Figure 1 Microscopic image of fetal liver showing intrasinusoidal development of hematopoietic cells; the predominant cell type is erythroid.

of hematopoietic stem cells, as described above) (Figure 1). Besides the yolk sac, hematopoietic stem cells are also produced from the aorta-gonadsmesonephros region. The aorta-gonads-mesonephros is an important transient site of development of stem cells, which populate the marrow. The bone marrow forms relatively late in embryogenesis, and establishment of hematopoietic elements in the medullary cavities of bone occurs at approximately 8 weeks of development. By 11 weeks, a stromal environment and hematopoietic cells are established in the marrow cavity and produce, primitive, but identifiable blood components. Spleen plays a minor role as a site of hematopoiesis, predominantly of erythroid elements. At each step of development, a complex interaction of local stroma, cell-cell interactions and the influence of cytokines and hematopoietic growth factors play a central role. ${ }^{3}$

\section{General, Benign EMH}

In general, there are three circumstances which underlie the abnormal, extramedullary proliferation of normal hematopoietic elements: (1) filtration, where immature cells are trapped by the spleen or other sites (such as in cases of acquired or functional hyposplenism) and proliferate; (2) inadequate marrow space to produce appropriate numbers of marrow elements (owing to compromise of marrow cavity, eg myelophthisis, or overproduction of marrow elements) or damage to the bone marrow microenvironment leading to increased numbers of circulating hematopoietic stem cells; ${ }^{4}(3)$ abnormal cytokine or other circulating hematopoietic growth factors causing stem cell differentiation to hematopoietic cells or local effects simulating the marrow microenvironment. ${ }^{1}$
The first cause, filtration, is mostly seen in marrow disorders with mobilization of increased numbers of immature hematopoietic precursor cells (such as chronic idiopathic myelofibrosis). The second circumstance, compromise of, or damage to the marrow space, can be caused by a variety of neoplastic and nonneoplastic and stromal disorders affecting the marrow. The third cause, generation of hematopoietic growth-stimulating factors, may occur independently, as a result of hematopoietic growth factors production by tumor or at sites of tissue damage and repair. It should be noted that although some cases may be caused by one of these factors, it is likely more than one factor contributes in most circumstances.

In a large study, Koch et $a l^{1}$ found that $78 \%$ of cases of nonhepatosplenic EMH were symptomatic. Pleural effusion, ascites, neurologic deficit, cardiac tamponade, chronic renal failure, acute respiratory failure, orbital proptosis, subglottic stenosis are among the symptoms associated with EMH.

Tissue biopsy, fine needle aspiration, radionucleotide scanning have all been used as diagnostic techniques to evaluate extramedullary myeloid disease. ${ }^{1}$ The radiologic appearance of myeloid sarcoma can be distinctive enough for a presumptive diagnosis. ${ }^{5}$

Localized irradiation, red blood cell transfusions, surgical excision, decompressive laminectomy and chemotherapy including hydroxyurea are most commonly used in therapy for extramedullary myeloid diseases. ${ }^{1}$ Localized therapy has no apparent effect on long-term outcomes, independent of systemic therapy. Jenkin et $a l^{6}$ recommended that localized irradiation should be limited to cases where there is imminent compromise or critical organ damage (eg eye, brain, spinal cord and kidney).

\section{Pediatric}

The finding of EMH in infants is not unusual. The most common sites are liver, spleen and lymph nodes, but EMH can be found in other sites as well (Figure 2). It is expected that as the infant develops in age, extramedullary sites would regress. Undoubtedly, premature births or a perinatal period associated with physiologic stress would have the potential for persistence of EMH. This EMH would serve the biologic purpose of producing more hematopoietic elements, in response to whatever pre- or perinatal stress was present. It is easy to conceive that a variety of causes would induce retention or expansion of the hematologic elements. These would include bleeding, infections, hypoxia and a wide variety of congenital defects of heart, lungs, kidneys and other organs and systems.

\section{Hematologic Disorders}

Benign hematologic disorders are a well-known cause of expansion of the hematopoietic-producing 

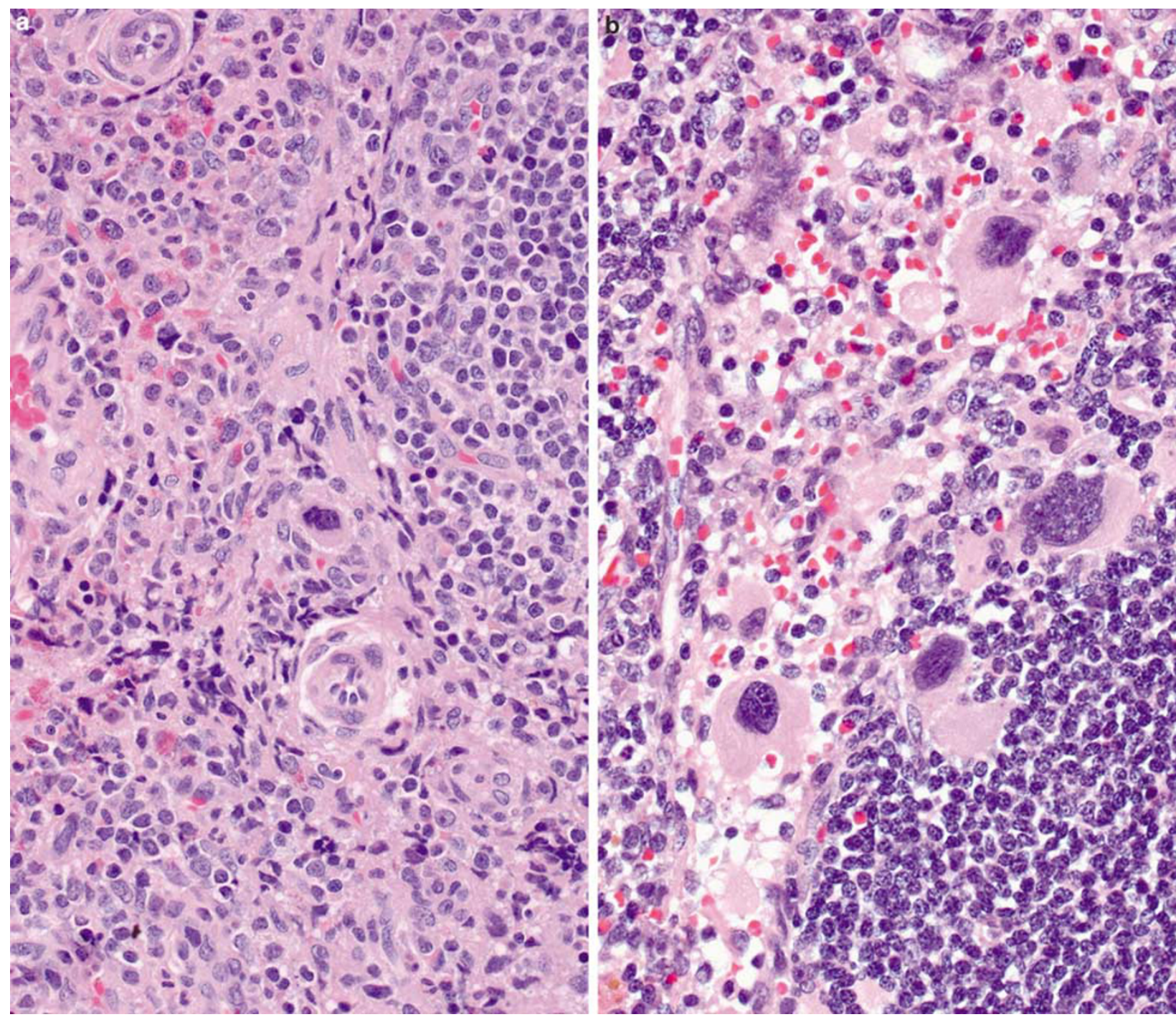

Figure 2 Myeloid proliferations in lymph node. (a) Image from an enlarged node in a 6-week-old premature infant. This benign proliferation consists of predominantly myeloid elements including numerous eosinophil precursors. (b) Enlarged lymph node from an adult with a long history of chronic idiopathic myelofibrosis. Under this circumstance the proliferation is neoplastic and the proliferation consists of dysplastic megakaryocytes and immature myeloid elements.

space beyond the confines of the marrow cavity. The most common of these disorders include thalassemia, hereditary spherocytosis, sickle cell anemia, congenital dyserythroblastic anemia and immune thrombocytopenic purpura. Red blood cell disorders are a relatively common cause of these findings. Defects in the red blood cell membrane, enzyme pathways, hemoglobin or other defects will cause reduced production, shortened lifespan or ineffective red blood cells. Because of the paucity of red blood cells, a natural homeostatic response is to increase the production, through a variety of homeostatic mechanisms. Most typically, hypoxia will activate the production of erythropoietin in the kidney. Erythropoietin drives the production of red blood cell precursors as well as proliferation and maturation.

Under some circumstances, this compensatory mechanism is adequate, and the only effect is a relative erythroid hyperplasia seen in the bone marrow. However, if the reduction of red blood cells or hypoxia is severe enough, then the available space in the marrow cavity will not be enough to meet the red blood cell production needs. Under these circumstances, marrow elements will be produced in extramedullary sites. Comparable conditions occur in which there are ineffective production of granulocytic or platelet/megakaryocytic elements. Expansion of the cells types are less common, but could also result in EMH.

\section{Stromal Disorders and Other Causes}

There are stromal disorders of the marrow that cause abnormalities of the marrow environment, which lead to proliferations of EMH. One such example is osteopetrosis, or 'marble-bone disease'. This is a 
disorder of abnormal, excess bone formation and proliferation. The marrow cavity itself is compromised, with little room for hematopoietic elements. As such, in order to survive, hematopoiesis must be shift to another, nonmedullary sites. In these patients EMH may be widespread, including the thoracic cavity. Similar changes have also been seen in Paget disease and severe cases of renal osteodystrophy.

Replacement of the marrow cavity space by nonhematopoietic tissue is referred to as myelophthisis. It can be due to a variety of factors, including bone disorders, as well as extensive marrow involvement by infectious disease (eg tuberculosis), storage diseases (ie Gaucher, Neimann-Pick) or metastatic tumor. Marrow fibrosis, in general, may induce EMH. The fibrosis is not itself a neoplastic process, and may be a result of a variety of nonneoplastic conditions. However, severe marrow fibrosis is most commonly associated with neoplasms, including myeloid, lymphoid and metastatic cancers.

The administration of hematopoietic growth factors, such as granulocyte colony-stimulating factor (G-CSF) and granulocyte/monocyte CSF (GM-CSF) have become common therapies for a variety of disorders. The predominant uses of G-CSF and GM-CSF are for white blood cell cytopenias and for mobilization of stem cells for collection. ${ }^{7,8} \mathrm{~A}$ rare, but not unanticipated consequence of administration of the potent hematopoietic growth factors is the induction of hematopoiesis in extramedullary sites. It is well recognized that treatment with G-CSF and GM-CSF causes increases in spleen size (Figure 3). ${ }^{9-11}$ This is because of the induction of EMH, which can under extreme circumstances cause splenic rupture. ${ }^{12,13}$ Hematopoietic cytokines

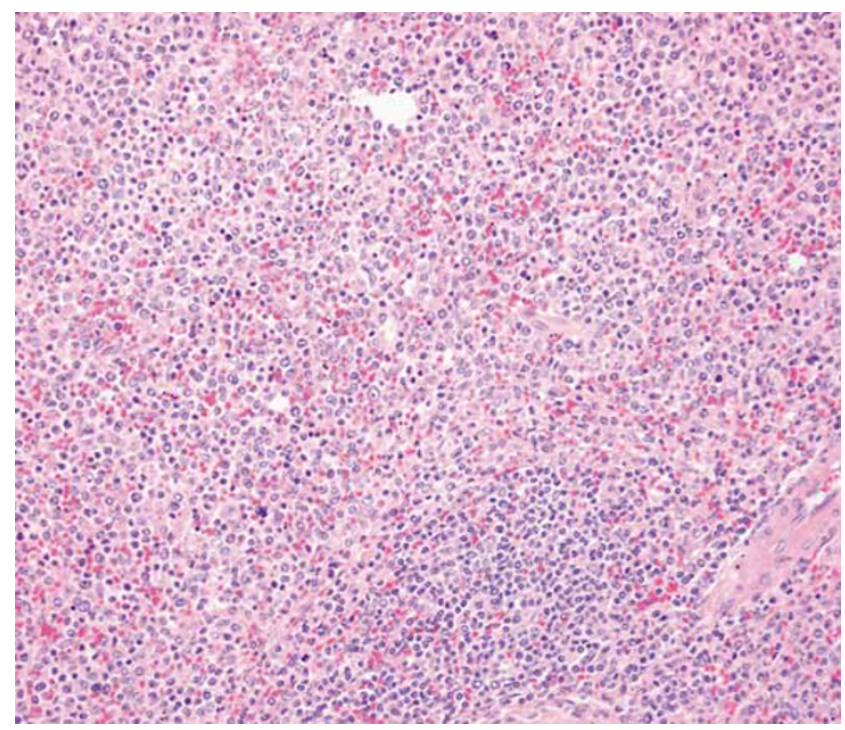

Figure 3 Splenic enlargement owing to granulocyte colonystimulating factor therapy. The red pulp is expanded by a massive proliferation of granulocytic precursors. have also been associated with EMH in other sites, including lymph nodes. ${ }^{14-16}$

\section{EMH in Spleen}

Spleen is a relatively frequent site of EMH. When seen in these sites, it can be attributed to several circumstances: persistence of fetal hematopoiesis (pediatric patients), in response to benign hematologic or stromal disorders, very small amounts of normal 'resident' hematopoiesis, or a NMP in association with a myeloid neoplasm (Figure 4).

As discussed earlier, the spleen plays only a minor role in the embryologic stages of hematopoiesis. Therefore, it is somewhat surprising that it is such a common site of benign marrow proliferations in postnatal life. The splenic microenvironment is relatively harsh and inhospitable, which is due to functional and physical arrangement of the spleen. The red pulp is a hypoxic, acidotic environment with numerous macrophages ready to destroy cellular or foreign elements that do not belong. This environment puts tremendous stress on red blood cells. Red blood cells that lack deformability or have abnormalities of surface membranes are destroyed by splenic macrophages and the components are recycled.

In spite of this inhospitable environment, marrow elements will proliferate in the spleen under a variety of conditions. Usually, the proliferation takes place within the red pulp, in the same areas that are acidotic and hypoxic. Marrow elements that become trapped in the spleen have some proliferative potential. ${ }^{4}$ In addition, the stroma, while not exactly the same as the bone marrow, could at least be considered 'permissive' for proliferation of marrow elements.

Several studies have shown extensive splenic EMH in a variety of nonneoplastic conditions including thrombotic thrombocytopenic purpura/ hemolytic uremic syndrome, in post-bone marrow transplant and in some immunodeficiencies. ${ }^{17,18} \mathrm{In}$ thrombotic thrombocytopenic purpura/hemolytic uremic syndrome consumption of platelets and red blood cells in the course of the disease process may induce hematopoietic stem cells to expand. However, splenomegaly, increases in myeloid elements, and the large increases in extent of EMH are not seen in other comparable conditions, and may represent a relatively unique, albeit rare, feature of thrombotic thrombocytopenic purpura/hemolytic uremic syndrome. In post-bone marrow transplant patients, the spleen serves as a site of hematopoietic reconstitution. Under both these conditions, damage to the marrow microenvironment may contribute to the presence of EMH. EMH can also be seen in several nonmyeloid neoplasms within the spleen including vascular neoplasms (benign and malignant), B-cell lymphomas and epithelial metastases. 

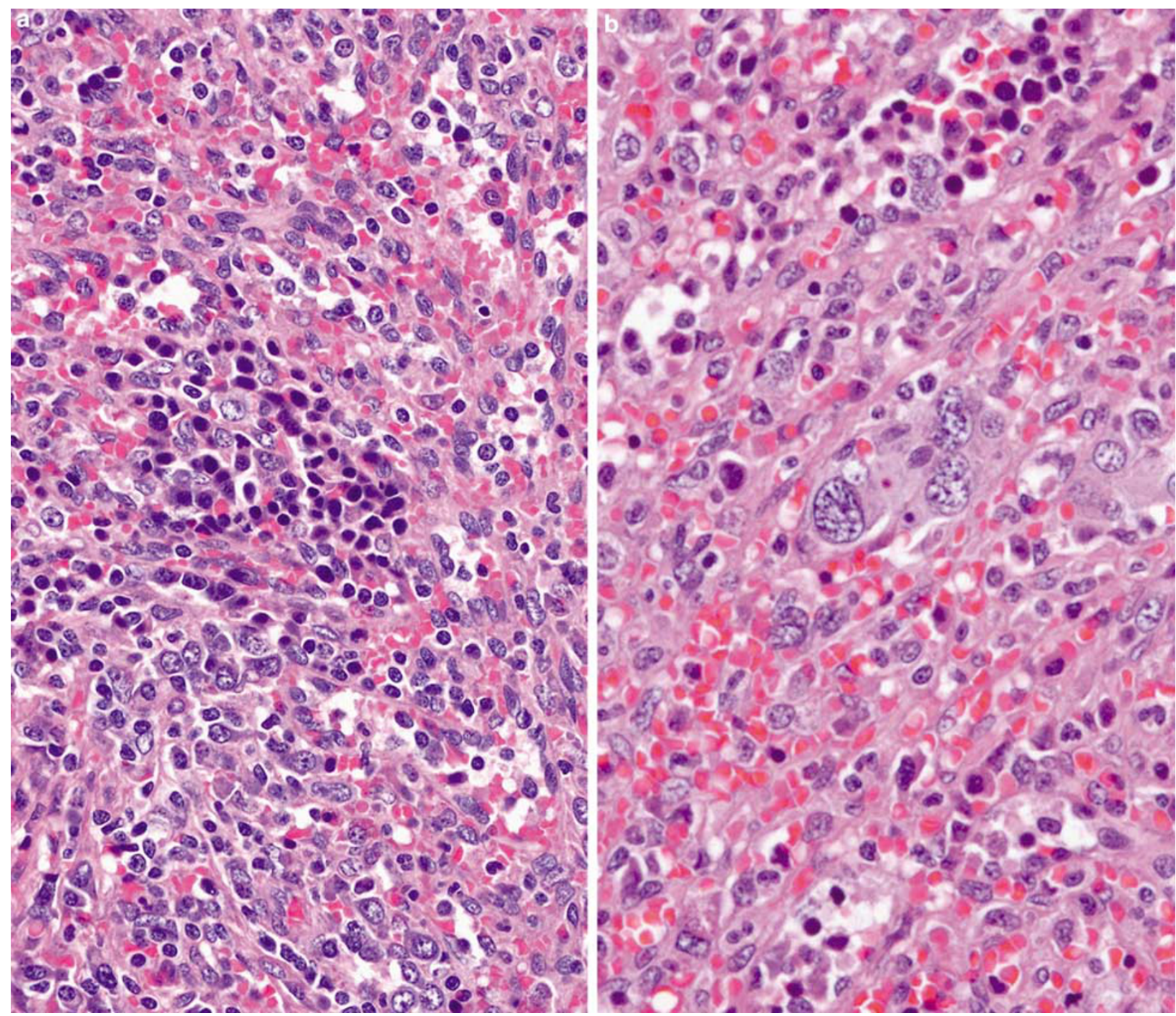

Figure 4 Splenic myeloid proliferations. (a) Splenic EMH consisting of erythroid precursors in this spleen of a patient with thalassemia. (b) Trilineage proliferation of marrow elements, including immature myeloid elements and dysplastic megakaryocytes in this patient with chronic idiopathic myelofibrosis.

\section{EMH in Lymph Node}

Under most circumstances, there are few times or locations where proliferation of marrow elements (myeloid, erythroid, megakaryocytic) in other than tiny amounts, would be considered normal in lymph nodes. In an adult, the presence of a marrow proliferation in a lymph node would indicate an immediate search for an underlying bone marrow neoplasm, such as a chronic myeloproliferative disorder. However, as in the spleen, lymph nodes may occasionally have proliferations of marrow elements. The presence of this EMH in lymph nodes is most often associated with the causes as described above, such as inherent hematologic disorders. In pediatric patients, finding EMH in a lymph node may suggest an as yet undiagnosed benign hematologic disorder, and adenopathy with EMH may be an initial manifestation. Regardless, in both adults and pediatric patients, the presence of any significant amount of EMH in a lymph node should prompt a hematologic evaluation, including review of a hemogram, peripheral blood smear and possibly a bone marrow aspiration and biopsy.

\section{EMH, Other Sites}

\section{Liver}

Since liver is a site for early hematopoiesis, it is not surprising that it is a relatively frequent site of EMH. In pediatric patients, EMH, predominantly erythroid, can be seen in association with a variety of liver diseases (hepatitis, etc) as well as hematologic disorders, as mentioned previously. It is typically located within the hepatic sinusoids. In pediatric patients, the presence of hepatic EMH is considered normal up to about 5 weeks of age. ${ }^{19}$ In adults, hepatic EMH is seen in a variety of conditions, including hematologic disorders, sepsis, transplantation, massive hepatic necrosis, but is also notably 
prominent within hepatoblastomas, hepatic adenomas and hepatocellular carcinoma. ${ }^{20-22}$ The cause of these proliferations is not well understood, and may be due to local hypoxic conditions, local effects of tumor that simulate the embryonic liver and are supportive of hematopoietic development, or production of growth factors that attract and/or support hematopoiesis. ${ }^{22}$ In hepatoblastomas, von Schweinitz et $a l^{23}$ showed that there was production of numerous hematopoietic growth factors (Figure 5).

\section{Heart}

A rare, but notable finding is the presence of EMH within heart biopsies or explanted specimens. Goldman and Wurzel ${ }^{24}$ noted EMH within hearts with recent myocardial infarctions; they did not see EMH in hearts with remote infarcts. Hill and Swanson ${ }^{25}$ found EMH in hearts of a wide age range of pediatric and adult patients, all with risk factors for cardiac ischemia, and a close association with the healing phase of myocardial infarction. The presence of EMH under these circumstances suggests that circulating stem cells are attracted to the site by cytokines. ${ }^{25}$

\section{Tumors}

$\mathrm{EMH}$ has been reported in association with several types of tumors including cerebellar hemangioblastoma, ${ }^{26-28}$ hemangiomas, ${ }^{29}$ hepatoblastomas, ${ }^{23,30}$ leiomyomas, ${ }^{31}$ pilomatricomas, ${ }^{32}$ hepatic angiosarcoma, ${ }^{33}$ endometrial carcinoma, ${ }^{34,35}$ meningioma, ${ }^{36}$ hepatic adenoma, ${ }^{37}$ spindle cell lipoma, ${ }^{38}$ liposarcoma, ${ }^{39}$ myofibroblastic tumors and renal tumors. ${ }^{25}$

\section{Myelolipoma}

A special circumstance of a benign myeloid proliferation that requires separate discussion is that of myelolipomas. Originally described by Gierke in $1905,{ }^{40}$ the term myelolipoma was first used by Oberling in 1929. ${ }^{41}$ Myelolipomas are rare tumors, composed of mature fat associated with hematopoietic elements, most commonly found in the adrenal gland,

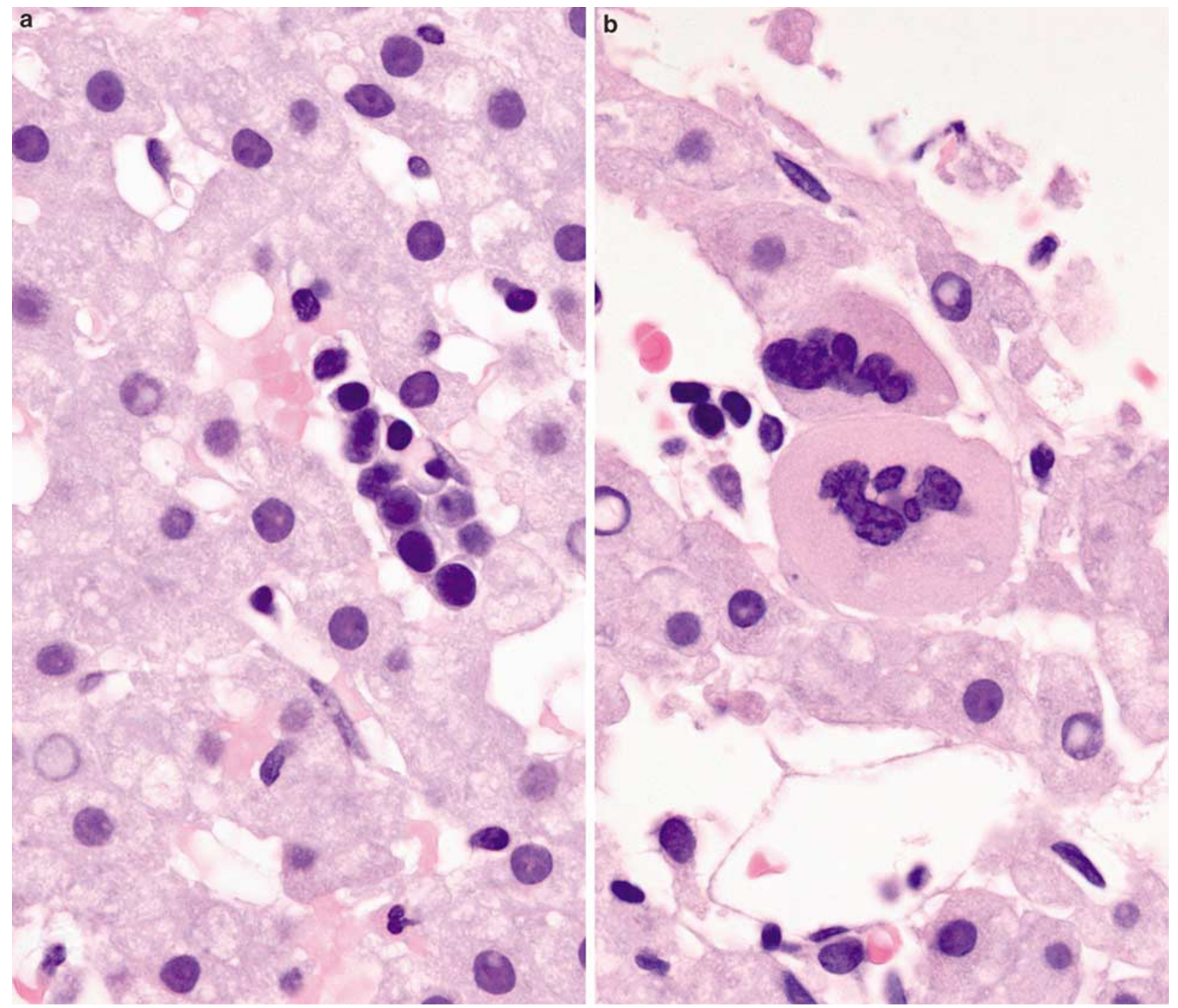

Figure 5 Examples of intrasinusoidal marrow elements in a hepatic adenoma. (a) Erythroid elements and (b) megakaryocytes. 
other retroperitoneal locations and rarely in the mediastinum or other sites (Figure 6). ${ }^{42-46}$ Myelolipomas are often asymptomatic and discovered incidentally in the course of radiologic evaluation of the abdomen. Rarely, they present with symptoms, usually due to large size and/or abdominal pain. ${ }^{42-46}$

A case report found a clonal cytogenetic abnormality in a myelolipoma, suggesting that they may be clonal proliferations. ${ }^{47}$ In addition, a recent study by Bishop et $a l^{48}$ showed $80 \%$ of myelolipomas analyzed by X-chromosome inactivation showed

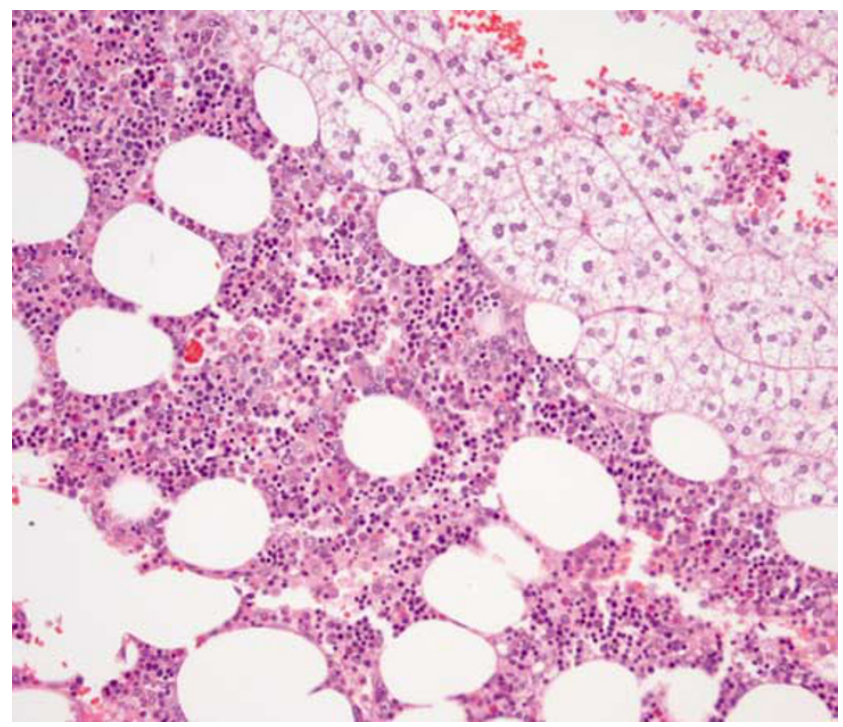

Figure 6 A microscopic image of a myelolipoma including adrenal cortex (upper right) and well-differentiated marrow elements and fat. nonrandom X-chromosome inactivation in both the marrow elements and fat. This finding suggests that in most cases myelolipomas are clonal and that the fat and myeloid elements are derived from a common precursor. At the present time, there have been no reports of malignant transformation of myeloid elements in a myelolipoma.

The study by Bishop et a $1^{48}$ also showed that myelolipomas, although similar to normal bone marrow, have significant variations in terms of cellular composition, cellularity and stromal composition. Although all of the myelolipomas studied demonstrated normal trilineage hematopoiesis, only very rare early myeloid precursors were present in these lesions.

\section{Differential Diagnosis}

When considering the differential diagnosis of EMH, extramedullary proliferations of neoplastic hematopoietic elements, is a primary consideration. In virtually all circumstances, a patient with a neoplastic extramedullary proliferation of myeloid elements would have systemic involvement (and presumably marrow involvement) by a myeloid neoplasm. Quite rarely, however, extramedullary disease may be the only site of involvement, or may occur as a localized antecedent to more generalized disease or as a site of recurrence.

Although historically the term 'EMH' has been used to describe these proliferations, this terminology is less than optimal, as it could suggest that these proliferations of myeloid elements are comparable to nonneoplastic proliferations discussed previously (Table 1). As such, the term NMP is

Table 1 Distinction of benign and neoplastic myeloid proliferations

\begin{tabular}{|c|c|c|}
\hline & Benign & Neoplastic \\
\hline Morphology & $\begin{array}{l}\text { Unilineage proliferation: most commonly } \\
\text { erythroid. Erythroid cells identifiable by } \\
\text { hyperchromatic round nucleus with clear or pink } \\
\text { ring of cytoplasm. Often present in clusters. } \\
\text { Megakaryocytes identifiable by distinctive } \\
\text { morphology; large size, multilobed nuclei, } \\
\text { large amounts of granular cytoplasm. } \\
\text { Multilineage proliferation: often all } \\
\text { developmental stages of hematopoietic cells } \\
\text { are seen, but predominantly mature forms. }\end{array}$ & $\begin{array}{l}\text { Unilineage proliferation: typically } \\
\text { monomorphous population of intermediate sized, } \\
\text { noncohesive hematopoietic cells. Immature } \\
\text { eosinophils are an important morphologic clue. } \\
\text { Multilineage proliferation: most often seen in } \\
\text { CMPD or MDS. Clusters of immature myeloid } \\
\text { cells (eg blasts) may be seen. Megakaryocytes may } \\
\text { be dysplastic; small or abnormally large size, } \\
\text { hyperchromatic nucleus, hyper- or hypolobated } \\
\text { nuclei, clustering. }\end{array}$ \\
\hline
\end{tabular}

Immunohistochemistry

$$
\text { Rare or no expression of CD34, CD117, TdT }
$$
No molecular or cytogenetic abnormalities
expected.
Diffuse positivity supports diagnosis of myeloid sarcoma, if other nonhematopoietic lesions or mastocytosis are not a diagnostic consideration. Expression of CD34 or CD117 in clusters may herald transformation of CMPD or MDS to blast phase or acute myeloid leukemia.

Presence of cytogenetic abnormalities by conventional karyotype or FISH support a diagnosis of neoplastic proliferation. Specific defects (eg t $(9 ; 22)$ in $\mathrm{CML}$ ) can confirm diagnosis.

CML, chronic myelogenous leukemia; CMPD, chronic myeloproliferative disorder; MDS, myelodysplastic syndrome; FISH, fluorescent in situ hybridization. 
suggested to refer to these proliferations of extramedullary neoplastic marrow elements.

Myeloid sarcoma is a mass composed of myeloblasts or other immature myeloid cells occurring in an extramedullary site (Figure 7$).{ }^{49}$ Myeloid sarcoma is an infrequent finding in adults $(2-3 \%)$, but can be considerably more common in pediatric patients (up to $30 \%$ in some series). ${ }^{5}$ Although specific situations exist in which the presence of myeloid sarcoma has little effect on outcome, in general it heralds a poor prognosis..$^{5,49-52}$ Sites of involvement vary depending on the exact underlying disorder, age of patient and the presentation (eg preceding or concurrent with systemic disease). The tumors consist of populations of blasts, but mixtures of cell types are seen in the more differentiated subtypes. The differentiated-type consists of promyelocytes and more mature granulocytic forms and would most frequently be confused with EMH. The cells have scant to abundant amounts of cytoplasm. Eosinophilic myelocytes are often a helpful diagnostic clue when evaluating NMP, and are rarely seen in EMH. ${ }^{53}$

In addition to myeloid sarcoma, NMP can be seen in association with myeloproliferative disorders, myelodysplastic syndromes, myelodysplastic/myeloproliferative disorders and other myeloid-derived malignancies. Under these conditions, the cellular proliferations can consist of trilineage marrow elements and closely mimic the appearance of benign EMH. Specific sites of involvement depend on the exact underlying disorder, age of the patient and the presentation (eg preceding or concurrent with systemic disease). In general, the most common sites, in order of decreasing frequency, are spleen, lymph node, skin, bone, small intestine, orbit, breast, cervix, nasal sinus, mediastinum and brain. ${ }^{5,54}$ Less common sites include pericardium/
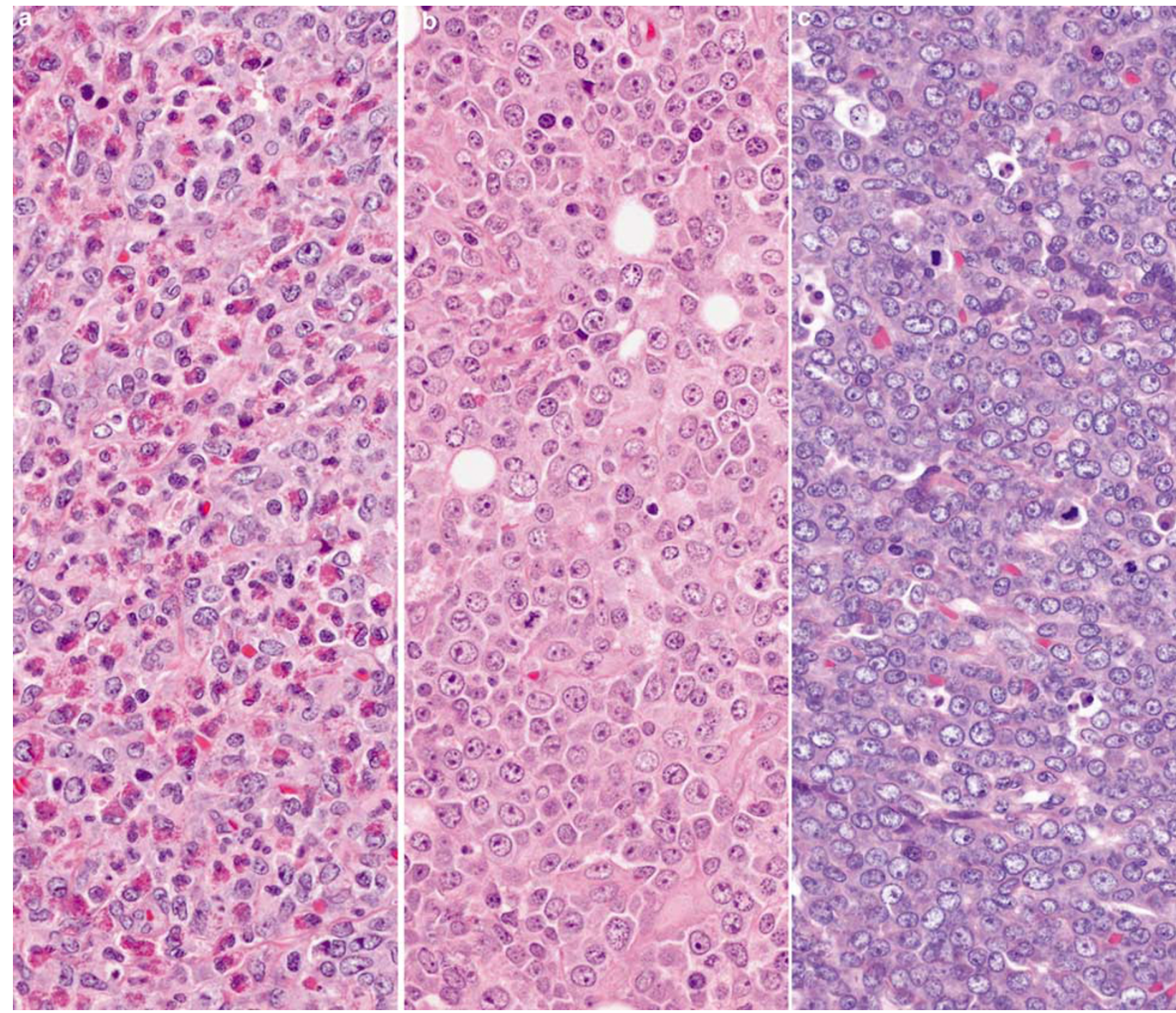

Figure 7 Examples of differing histologic appearance of myeloid sarcomas. (a) An example of the differentiated type of myeloid sarcoma, which has numerous eosinophils and more mature myeloid elements. (b) and (c) Consist of predominantly blastic cells, and would not typically be confused with EMH. Each is an intermediate-sized proliferation of cells that can be recognized as being of hematopoietic origin. 
heart, pleura/lung, uterus, testis and oral mucosa.

In evaluating EMH vs NMP, clinical history is of paramount importance. Any previous or current history of a myeloid neoplasm strongly supports the neoplastic nature of the proliferation. However, strictly speaking, the morphologic appearance can be a clue to the diagnosis, but cannot be completely relied upon. The presence of sheets or clusters of immature myeloid cells, eosinophilic precursors or markedly dysplastic megakaryocytes would be supportive of the neoplastic nature of a myeloid proliferation. However, supportive information, such as clinical history, bone marrow or peripheral blood findings, immunohistochemistry or molecular/genetic testing are required to resolve the nature of the most challenging cases.

\section{Immunohistochemical Studies}

Immunohistochemical stains are of benefit in positively identifying EMH. Both myeloperoxidase and lysozyme are both very specific in identifying myeloid/granulocytic elements. ${ }^{2,53}$ Other stains that are useful for immunohistochemical analysis include various forms of CD68, most notably CD68R (PGM-1) and CD68 (KP-1), HAM-56, elastase, CD34, CD117, CD15, CD14, CD163. ${ }^{2}$ CD34 expression is associated with an immature myeloid phenotype, but can be seen in other cellular lineages, most notably acute lymphoblastic leukemia. CD117 is quite a useful stain in the evaluation of myeloid neoplasms. In extramedullary myeloid proliferations, the presence of CD117 staining suggests a myeloid origin and the presence of immature myeloid cells. ${ }^{55}$

Monocytic lineage can be confirmed by a number of stains including CD163, CD14, CD68R (PGM-1) and HAM-56, which are seen in mature monocyte/ macrophages, and are only seen in a small subset of immature neoplasms. CD68 (KP-1) is quite nonspecific and can be seen in a variety of hematopoietic neoplasms. The presence of TdT is strongly supportive of acute lymphoblastic leukemia/lymphoma or myeloid sarcoma.

Identification of erythroid and megakaryocytic elements may occasionally useful, although these cells are typically more easily identifiable based on morphology alone. For erythroid elements, immunohistochemical stains for hemoglobin or glycophorin may be used for identification. Cells of megakaryocytic lineages may be identified by their positivity for CD41, CD42b or CD61. Other stains that are positive in megakaryocytes, but lack specificity include CD31, Factor VIII antigen, CD79a and antilinker of activated T-cells (anti-LAT). ${ }^{56}$

\section{Conclusion}

There are several circumstances that account for proliferation of marrow elements in extramedullary sites. The finding of EMH as a benign proliferation in response to a variety of benign conditions, either owing to excessive colonization of extramedullary sites by hematopoietic elements or growth factors are well described. Beside negative effects as space occupying lesions, they have little clinical consequence. Pathologists need to be familiar with their existence to not over interpret their clinical significance. Awareness of the differential diagnosis of EMH from NMPs is important to avoid overdiagnosing this benign condition.

\section{Acknowledgements}

I thank John Eble and Richard Neiman for their helpful comments on this manuscript.

\section{References}

1 Koch CA, Li CY, Mesa RA, et al. Nonhepatosplenic extramedullary hematopoiesis: associated diseases, pathology, clinical course, and treatment. Mayo Clin Proc 2003;78:1223-1233.

2 Quintanilla-Martinez L, Zukerberg LR, Ferry JA, et al. Extramedullary tumors of lymphoid or myeloid blasts. The role of immunohistology in diagnosis and classification. Am J Clin Pathol 1995;104:431-443.

3 Tavian M, Peault B. The changing cellular environments of hematopoiesis in human development in utero. Exp Hematol 2005;33:1062-1069.

4 Wolf BC, Neiman RS. Hypothesis: splenic filtration and the pathogenesis of extramedullary hematopoiesis in agnogenic myeloid metaplasia. Hematol Pathol 1987;1:77-80.

5 Byrd JC, Edenfield WJ, Shields DJ, et al. Extramedullary myeloid cell tumors in acute nonlymphocytic leukemia: a clinical review. J Clin Oncol 1995;13: 1800-1816.

6 Jenkin RD, Al-Shabanah M, Al-Nasser A, et al. Extramedullary myeloid tumors in children: the limited value of local treatment. J Pediatr Hematol Oncol 2000;22:34-40.

7 Takeyama K, Ohto H. PBSC mobilization. Transfus Apher Sci 2004;31:233-243.

8 Cashen AF, Link D, Devine S, et al. Cytokines and stem cell mobilization for autologous and allogeneic transplantation. Curr Hematol Rep 2004;3:406-412.

9 Platzbecker U, Prange-Krex G, Bornhauser M, et al. Spleen enlargement in healthy donors during G-CSF mobilization of PBPCs. Transfusion 2001;41:184-189.

10 Stroncek D, Shawker T, Follmann D, et al. G-CSFinduced spleen size changes in peripheral blood progenitor cell donors. Transfusion 2003;43:609-613.

11 Picardi M, De Rosa G, Selleri C, et al. Spleen enlargement following recombinant human granulocyte colony-stimulating factor administration for peripheral blood stem cell mobilization. Haematologica 2003;88:794-800.

12 Balaguer H, Galmes A, Ventayol G, et al. Splenic rupture after granulocyte-colony-stimulating factor mobilization in a peripheral blood progenitor cell donor. Transfusion 2004;44:1260-1261.

13 Nuamah NM, Goker H, Kilic YA, et al. Spontaneous splenic rupture in a healthy allogeneic donor of 
peripheral-blood stem cell following the administration of granulocyte colony-stimulating factor (G-CSF). A case report and review of the literature. Haematologica 2006;91:ECR08.

14 Lindemann A, Herrmann F, Mertelsmann R, et al. Splenic hematopoiesis following GM-CSF therapy in a patient with hairy cell leukemia. Leukemia 1990;4: 606-607.

15 Redmond III J, Kantor RS, Auerbach HE, et al. Extramedullary hematopoiesis during therapy with granulocyte colony-stimulating factor. Arch Pathol Lab Med 1994;118:1014-1015.

16 Friedman HD, Sanderson SO, Stein CK, et al. Extramedullary granulopoiesis mimicking recurrent lymphoma after prolonged administration of human recombinant granulocyte colony-stimulating factor. Ann Hematol 1998;77:79-83.

17 Saracco SM, Farhi DC. Splenic pathology in thrombotic thrombocytopenic purpura. Am J Surg Pathol 1990; 14:223-229.

18 O’Malley DP, Kim YS, Perkins S, et al. Comparison of splenic extramedullary hematopoiesis in benign and neoplastic hematopoietic disorders. Mod Pathol 2005; 18:1550-1561.

19 MacSween RNM, Burt AD, Portmann BC, et al. Pathology of the Liver, 4th edn. Churchill Livingstone: Edinburgh, 2002, pp 850-851.

20 Schlitt HJ, Schafers S, Deiwick A, et al. Extramedullary erythropoiesis in human liver grafts. Hepatology 1995;21:689-696.

21 Tsamandas AC, Jain AB, Raikow RB, et al. Extramedullary hematopoiesis in the allograft liver. Mod Pathol 1995;8:671-674.

22 Craig CE, Quaglia A, Dhillon AP. Extramedullary haematopoiesis in massive hepatic necrosis. Histopathology 2004;45:518-525.

23 von Schweinitz D, Schmidt D, Fuchs J, et al. Extramedullary hematopoiesis and intratumoral production of cytokines in childhood hepatoblastoma. Pediatr Res 1995;38:555-563.

24 Goldman BI, Wurzel J. Hematopoiesis/erythropoiesis in myocardial infarcts. Mod Pathol 2001;14:589-594.

25 Hill DA, Swanson PE. Myocardial extramedullary hematopoiesis: a clinicopathologic study. Mod Pathol 2000;13:779-787.

26 Zec N, Cera P, Towfighi J. Extramedullary hematopoiesis in cerebellar hemangioblastoma. Neurosurgery 1991;29:34-37.

27 Chishti MK, Bannister CM. Foci of extramedullary haemopoiesis in a cerebellar haemangioblastoma. Br J Neurosurg 1992;6:157-162.

28 Svensson AM, Pang Y, Moore NJ, et al. Cystic tumor of the cerebellum with megaloblastic erythropoiesis. Hemangioblastoma with megaloblastic hematopoiesis. Arch Pathol Lab Med 2006;130:886-889.

29 Green LK, Klima M, Burns TR. Extramedullary hematopoiesis occurring in a hemangioma of the skin. Arch Dermatol 1988;124:1720-1721.

30 Ishak KG, Glunz PR. Hepatoblastoma and hepatocarcinoma in infancy and childhood. Report of 47 cases. Cancer 1967;20:396-422.

31 Schmid C, Beham A, Kratochvil P. Haematopoiesis in a degenerating uterine leiomyoma. Arch Gynecol Obstet 1990;248:81-86.

32 Kaddu S, Beham-Schmid C, Soyer HP, et al. Extramedullary hematopoiesis in pilomatricomas. Am J Dermatopathol 1995;17:126-130.
33 Kojiro M, Nakashima T, Ito Y, et al. Thorium dioxiderelated angiosarcoma of the liver. Pathomorphologic study of 29 autopsy cases. Arch Pathol Lab Med 1985;109:853-857.

34 Sirgi KE, Swanson PE, Gersell DJ. Extramedullary hematopoiesis in the endometrium. Report of four cases and review of the literature. Am J Clin Pathol 1994;101:643-646.

35 Valeri RM, Ibrahim N, Sheaff MT. Extramedullary hematopoiesis in the endometrium. Int J Gynecol Pathol 2002;21:178-181.

36 Gregorios JB, Bay JW, Dudley Jr AW. Extramedullary hematopoiesis in a malignant meningioma. Neurosurgery 1983;13:447-451.

37 Wheeler DA, Edmondson HA, Reynolds TB. Spontaneous liver cell adenoma in children. Am J Clin Pathol 1986;85:6-12.

38 Sciot R, Bekaert J. Spindle cell lipoma with extramedullary haematopoiesis. Histopathology 2001;39: 215-216.

39 Lewis DJ, Moul JW, Williams SC, et al. Perirenal liposarcoma containing extramedullary hematopoiesis associated with renal cell carcinoma. Urology 1994;43: 106-109.

40 Gierke E. Uber Knochenmarksgewebe in der Nebenniere. Bietr Z Path Anat 1905;37:311.

41 Oberling C. Les formations myelolipomaleuses. Bull Assoc Fr Cancer 1929;18:234-236.

42 McDonnell WV. Myelolipomas of the adrenal. Arch Path 1956;61:416-419.

43 Plaut A. Myelolipoma in the adrenal cortex. Am J Pathol 1958;34:487-515.

44 Olsson CA, Krane RJ, Klugo RC, et al. Adrenal myelolipoma. Surgery 1973;73:665-670.

45 Sanders R, Bissada N, Curry N, et al. Clinical spectrum of adrenal myelolipoma: analysis of 8 tumors in 7 patients. J Urol 1995;153:1791-1793.

46 Lam KY, Lo CY. Adrenal lipomatous tumours: a 30 year clinicopathological experience at a single institution. J Clin Pathol 2001;54:707-712.

47 Chang K, Chen P, Huang Z, et al. Adrenal myelolipoma with translocation $(3 ; 21)(\mathrm{q} 25 ; \mathrm{p} 11)$. Cancer Genet Cytogenet 2002;134:77-80.

48 Bishop E, Eble JN, Cheng L, et al. Adrenal myelolipomas show non-random X-chromosome inactivation in hematopoietic elements and fat: Support for a clonal origin of myelolipomas. Am J Surg Pathol 2006;30: 838-843.

49 Brunning RD, Matutes E, Flandrin G, et al. Acute myeloid leukemia not otherwise categorized. In: Jaffe ES, Harris NL, Stein H, Vardiman JW (eds). Tumours of Haematopoietic and Lymphoid Tissues. IARC Press: Lyon, France, 2001, pp 91-105.

50 Dusenbery KE, Howells WB, Arthur DC, et al. Extramedullary leukemia in children with newly diagnosed acute myeloid leukemia: a report from the Children's Cancer Group. J Pediatr Hematol Oncol 2003;25: 760-768.

51 Byrd JC, Weiss RB, Arthur DC, et al. Extramedullary leukemia adversely affects hematologic complete remission rate and overall survival in patients with $\mathrm{t}(8 ; 21)(\mathrm{q} 22 ; \mathrm{q} 22)$ : results from Cancer and Leukemia Group B 8461. J Clin Oncol 1997;15:466-475.

52 Ginsberg JP, Orudjev E, Bunin N, et al. Isolated extramedullary relapse in acute myeloid leukemia: a retrospective analysis. Med Pediatr Oncol 2002; 38:387-390. 
53 Roth MJ, Medeiros LJ, Elenitoba-Johnson K, et al. Extramedullary myeloid cell tumors. An immunohistochemical study of 29 cases using routinely fixed and processed paraffin-embedded tissue sections. Arch Pathol Lab Med 1995; 119:790-798.

54 Neiman RS, Barcos M, Berard C, et al. Granulocytic sarcoma: a clinicopathologic study of 61 biopsied cases. Cancer 1981;48:1426-1437.
55 Chen J, Yanuck III RR, Abbondanzo SL, et al. c-Kit (CD117) reactivity in extramedullary myeloid tumor/ granulocytic sarcoma. Arch Pathol Lab Med 2001;125: 1448-1452.

56 Orazi A, O'Malley DP, Arber DA. The normal bone marrow and an approach to bone marrow evaluation of neoplastic and proliferative processes. In: Illustrated Pathology of the Bone Marrow. Cambridge University Press: Cambridge, 2006, pp 5-15. 